\title{
On new general integral inequalities for $h$-convex functions
}

Imdat Iscan ${ }^{1}$ and Mustafa Aydin ${ }^{2}$

${ }^{1}$ Department of Mathematics, Faculty of Arts and Sciences, Giresun University, Giresun, Turkey.

${ }^{2}$ Department of Finance-Banking and Insurance, Alucra Turan Barutçu Vocational School, Giresun University, Alucra-Giresun, Turkey.

Received: 25 December 2015, Accepted: 3 April 2016

Published online: 19 June 2016.

\begin{abstract}
In this paper, we derive new estimates for the remainder term of the midpoint, trapezoid, and Simpson formulae for functions whose derivatives in absolute value at certain power are $h$-convex and $h$-concave by using power mean inequality, Hölder inequality and some other integral inequalities. Some applications to special means of real numbers are also given.
\end{abstract}

Keywords: $h$-convex function, $h$-concave function, Simpson's inequality, Hermite-Hadamard's inequality.

\section{Introduction}

Let $f: I \subseteq \mathbb{R} \rightarrow \mathbb{R}$ be a convex function defined on the interval $I$ of real numbers and $a, b \in I$ with $a<b$. The following inequality

$$
f\left(\frac{a+b}{2}\right) \leq \frac{1}{b-a} \int_{a}^{b} f(x) d x \leq \frac{f(a)+f(b)}{2} .
$$

holds. This double inequality is known in the literature as Hermite-Hadamard integral inequality for convex functions. See $[1,2,4,7,8,9,11,12,13]$ for the results of the generalization, improvement and extention of the famous integral inequality (1).

In the paper [15] a large class of non-negative functions, the so-called $h$-convex functions is considered. This class contains several well-known classes of functions such as non-negative convex functions, $s$-convex in the second sense, Godunova Levin functions and $P$-functions. Let us recall definitions of these special classes of functions.

Definition 1. $f: I \rightarrow R$ is a Godunova-Levin function or that $f$ belongs to the class $Q(I)$ if $f$ is non-negative and for all $x, y \in I$ and $\alpha \in(0,1)$ we have

$$
f(\alpha x+(1-\alpha) y) \leq \frac{f(x)}{\alpha}+\frac{f(y)}{1-\alpha}
$$

The class $Q(I)$ was firstly described in [5] by Godunova and Levin. We note that non-negative monotone and non-negative convex functions belong to this class of functions.

In 1978, Breckner introduced $s$-convex functions as a generalization of convex functions as follows [3]: 
Definition 2. Let $s \in(0,1]$ be a fixed real number. A function $f:[0, \infty) \rightarrow[0, \infty)$ is said to be s-convex (in the second sense),or that $f$ belongs to the class $K_{s}^{2}$, if

$$
f(\alpha x+(1-\alpha) y) \leq \alpha^{s} f(x)+(1-\alpha)^{s} f(y)
$$

for all $x, y \in[0, \infty)$ and $\alpha \in[0,1]$.

Of course, $s$-convexity means just convexity when $s=1$. In [4] Dragomir et al. defined the concept of $P$-function as the following:

Definition 3. We say that $f: I \rightarrow \mathbb{R}$ is a P-function, or that $f$ belongs to the class $P(I)$, if $f$ is a non-negative function and for all $x, y \in I, \alpha \in[0,1]$, we have

$$
f(\alpha x+(1-\alpha) y) \leq f(x)+f(y)
$$

Let $I$ and $J$ be intervals in $\mathbb{R},(0,1) \subseteq J$ and $h$ and $f$ be real non-negative functions defined on $J$ and $I$, respectively. In [15], Varošanec defined the concept of $h$-convexity as follows:

Definition 4. Let $h: J \rightarrow \mathbb{R}$ be a non-negative function, $h \neq 0$. We say that $f: I \subseteq \mathbb{R} \rightarrow \mathbb{R}$ is an $h$-convex function or that $f$ belongsto the class $S X(h, I)$, if $f$ is non-negative function and for all $x, y \in I$ and $\alpha \in(0,1)$ we have

$$
f(\alpha x+(1-\alpha) y) \leq h(\alpha) f(x)+h(1-\alpha) f(y)
$$

If inequality (2) is reversed, then $f$ is said to be $h$-concave, i.e. $f \in S V(h, I)$. The notion of $h$-convexity unifies and generalizes the known classes of convex functions, $s$-convex functions, Gudunova-Levin functions and $P$-functions, which are obtained by putting in (2), $h(t)=t, h(t)=t^{s}, h(t)=\frac{1}{t}$, and $h(t)=1$, respectively. For some recent results about $h$ convex functions we refer the reader to papers $[2,10,13,14]$.

Remark.[15] Let $h$ be a nonnegative function such that

$$
h(\alpha) \geq \alpha
$$

for all $\alpha \in(0,1)$. For example, the function $h_{k}(x)=x^{k}$, where $k \leq 1$ and $x>0$, has that property. If $f$ is a nonnegative convex function on $I$, then for $x, y \in I, \alpha \in(0,1)$, we have

$$
f(\alpha x+(1-\alpha) y) \leq \alpha f(x)+(1-\alpha) f(y) \leq h(\alpha) f(x)+h(1-\alpha) f(y)
$$

So, $f \in S X(h, I)$. Similarly, if the function $h$ has the property $h(\alpha) \leq \alpha$ for all $\alpha \in(0,1)$, then any nonnegative concave function $f$ belongs to the class $S V(h, I)$.

In [13], Sarikaya et. al. established a new Hadamard-type inequality for $h$-convex functions.

Theorem 1. Let $f \in S X(h, I), a, b \in I$ with $a<b$ and $f \in L([a, b])$. Then

$$
\frac{1}{2 h\left(\frac{1}{2}\right)} f\left(\frac{a+b}{2}\right) \leq \frac{1}{b-a} \int_{a}^{b} f(x) d x \leq[f(a)+f(b)] \int_{0}^{1} h(t) d t .
$$

The following inequality is well known in the literature as Simpson's inequality. 
Let $f:[a, b] \rightarrow \mathbb{R}$ be a four times continuously differentiable mapping on $(a, b)$ and $\left\|f^{(4)}\right\|_{\infty}=\sup _{x \in(a, b)}\left|f^{(4)}(x)\right|<\infty$. Then the following inequality holds:

$$
\left|\frac{1}{3}\left[\frac{f(a)+f(b)}{2}+2 f\left(\frac{a+b}{2}\right)\right]-\frac{1}{b-a} \int_{a}^{b} f(x) d x\right| \leq \frac{1}{2880}\left\|f^{(4)}\right\|_{\infty}(b-a)^{4} .
$$

In [10], İşcan derived some new integral inequalities for functions with $h$-convex and $h$-concave first derivatives as follow:

Theorem 2. Let $f: I \subseteq \mathbb{R} \rightarrow \mathbb{R}$ be a differentiable mapping on $I^{\circ}$ such that $f^{\prime} \in L[a, b]$, where $a, b \in I^{\circ}$ with $a<b$ and $\theta, \lambda \in[0,1]$. If $\left|f^{\prime}\right|^{q}$ is h-convex on $[a, b], q>1$, then the following inequality holds:

$$
\begin{aligned}
& \left|(1-\theta)(\lambda f(a)+(1-\lambda) f(b))+\theta f((1-\lambda) a+\lambda b)-\frac{1}{b-a} \int_{a}^{b} f(x) d x\right| \\
& \leq(b-a)\left(\frac{\theta^{p+1}+(1-\theta)^{p+1}}{p+1}\right)^{\frac{1}{p}}\left(\int_{0}^{1} h(t) d t\right)^{\frac{1}{q}} \\
& \times\left[\lambda^{2}\left(\left|f^{\prime}(a)\right|^{q}+\left|f^{\prime}(C)\right|^{q}\right)^{\frac{1}{q}}+(1-\lambda)^{2}\left(\left|f^{\prime}(b)\right|^{q}+\left|f^{\prime}(C)\right|^{q}\right)^{\frac{1}{q}}\right]
\end{aligned}
$$

where $C=(1-\lambda) a+\lambda b$ and $\frac{1}{p}+\frac{1}{q}=1$.

In Theorem 2 , if we take $\lambda=1 / 2$, we have the following inequality

$$
\begin{aligned}
& \left|(1-\theta)\left(\frac{f(a)+f(b)}{2}\right)+\theta f\left(\frac{a+b}{2}\right)-\frac{1}{b-a} \int_{a}^{b} f(x) d x\right| \\
& \leq \frac{(b-a)}{4}\left(\frac{\theta^{p+1}+(1-\theta)^{p+1}}{p+1}\right)^{\frac{1}{p}}\left(\int_{0}^{1} h(t) d t\right)^{\frac{1}{q}} \\
& \times\left[\left(\left|f^{\prime}(a)\right|^{q}+\left|f^{\prime}\left(\frac{a+b}{2}\right)\right|^{q}\right)^{\frac{1}{q}}+\left(\left|f^{\prime}(b)\right|^{q}+\left|f^{\prime}\left(\frac{a+b}{2}\right)\right|^{q}\right)^{\frac{1}{q}}\right] .
\end{aligned}
$$

Theorem 3. Let $f: I \subseteq \mathbb{R} \rightarrow \mathbb{R}$ be a differentiable mapping on $I^{\circ}$ such that $f^{\prime} \in L[a, b]$, where $a, b \in I^{\circ}$ with $a<b$ and $\alpha, \lambda \in[0,1]$. If $\left|f^{\prime}\right|^{q}$ is $h$-concave on $[a, b], q>1$, then the following inequality holds:

$$
\begin{aligned}
& \left|S_{f}(\lambda, \theta, a, b)\right| \\
& \leq(b-a)\left(\frac{1}{2 h\left(\frac{1}{2}\right)}\right)^{\frac{1}{q}}\left(\frac{\theta^{p+1}+(1-\theta)^{p+1}}{p+1}\right)^{\frac{1}{p}} \\
& \times\left\{\lambda^{2}\left|f^{\prime}\left(\frac{(2-\lambda) a+\lambda b}{2}\right)\right|+(1-\lambda)^{2}\left|f^{\prime}\left(\frac{(1-\lambda) a+(1+\lambda) b}{2}\right)\right|\right\},
\end{aligned}
$$

where $C=(1-\lambda) a+\lambda b$ and $1 / p+1 / q=1$. 
In Theorem 3 , if we take $\lambda=1 / 2$, we have the following inequality

$$
\begin{aligned}
& \left|(1-\theta)\left(\frac{f(a)+f(b)}{2}\right)+\theta f\left(\frac{a+b}{2}\right)-\frac{1}{b-a} \int_{a}^{b} f(x) d x\right| \\
& \leq \frac{(b-a)}{4}\left(\frac{1}{2 h\left(\frac{1}{2}\right)}\right)^{\frac{1}{q}}\left(\frac{\theta^{p+1}+(1-\theta)^{p+1}}{p+1}\right)^{\frac{1}{p}} \\
& \times\left\{\left|f^{\prime}\left(\frac{3 a+b}{4}\right)\right|+\left|f^{\prime}\left(\frac{3 b+a}{4}\right)\right|\right\} .
\end{aligned}
$$

In [7], İşcan obtained a new generalization of some integral inequalities for differentiable convex mapping which are connected Simpson and Hadamard type inequalities, and he used the following lemma to prove this.

Lemma 1. Let $f: I \subseteq \mathbb{R} \rightarrow \mathbb{R}$ be a differentiable mapping on $I^{\circ}$ such that $f^{\prime} \in L[a, b]$, where $a, b \in I$ with $a<b$ and $\alpha, \lambda \in[0,1]$. Then the following equality holds:

$$
\begin{aligned}
& \lambda(\alpha f(a)+(1-\alpha) f(b))+(1-\lambda) f(\alpha a+(1-\alpha) b)-\frac{1}{b-a} \int_{a}^{b} f(x) d x \\
& =(b-a)\left[\int_{0}^{1-\alpha}(t-\alpha \lambda) f^{\prime}(t b+(1-t) a) d t+\int_{1-\alpha}^{1}(t-1+\lambda(1-\alpha)) f^{\prime}(t b+(1-t) a) d t\right] .
\end{aligned}
$$

In this paper, we will establish some new integral inequalities of Hermite-Hadamard and Simpson type for $h$-convex and $h$-concave functions using Lemma 1.

\section{Main results}

Let $f: I \subseteq \mathbb{R} \rightarrow \mathbb{R}$ be a differentiable function on $I^{\circ}$, the interior of $I$, throughout this section we will take

$$
I_{f}(\lambda, \alpha, a, b)=\lambda(\alpha f(a)+(1-\alpha) f(b))+(1-\lambda) f(\alpha a+(1-\alpha) b)-\frac{1}{b-a} \int_{a}^{b} f(x) d x
$$

where $a, b \in I^{\circ}$ with $a<b$ and $\alpha, \lambda \in[0,1]$.

The following theorem give a new result of integral inequalities for $h$-convex functions. In the sequel of the paper $I$ and $J$ are intervals in $\mathbb{R},(0,1) \subset J$ and $h$ and $f$ are real non-negative functions defined on $J$ and $I$, respectively and $h$ $\in L[0,1], h \neq 0$.

Theorem 4. Let $f: I \subseteq \mathbb{R} \rightarrow \mathbb{R}$ be a differentiable mapping on $I^{\circ}$ such that $f^{\prime} \in L[a, b]$, where $a, b \in I^{\circ}$ with $a<b$ and $\alpha, \lambda \in[0,1]$. If $\left|f^{\prime}\right|^{q}$ is h-convex on $[a, b], q \geq 1$, then the following inequality holds:

$$
\left|I_{f}(\lambda, \alpha, a, b)\right| \leq\left\{\begin{array}{l}
(b-a)\left[\gamma_{2}^{1-\frac{1}{q}} A^{\frac{1}{q}}+v_{2}^{1-\frac{1}{q}} B^{\frac{1}{q}}\right], \alpha \lambda \leq 1-\alpha \leq 1-\lambda(1-\alpha) \\
(b-a)\left[\gamma_{2}^{1-\frac{1}{q}} A^{\frac{1}{q}}+v_{1}^{1-\frac{1}{q}} B^{\frac{1}{q}}\right. \\
(b-a)\left[\gamma_{1}^{1-\frac{1}{q}} A^{\frac{1}{q}}+v_{2}^{1-\frac{1}{q}} B^{\frac{1}{q}}\right], \alpha \lambda \leq 1-\lambda(1-\alpha) \leq 1-\alpha
\end{array}\right.
$$


where

$$
\begin{gathered}
\gamma_{1}=\gamma_{1}(\alpha, \lambda)=(1-\alpha)\left[\alpha \lambda-\frac{(1-\alpha)}{2}\right], \\
\gamma_{2}=\gamma_{2}(\alpha, \lambda)=(\alpha \lambda)^{2}-\gamma_{1}(\alpha, \lambda), \\
v_{1}=v_{1}(\alpha, \lambda)=\frac{1-(1-\alpha)^{2}}{2}-\alpha[1-\lambda(1-\alpha)], \\
v_{2}=v_{2}(\alpha, \lambda)=\frac{1+(1-\alpha)^{2}}{2}-(\lambda+1)(1-\alpha)[1-\lambda(1-\alpha)], \\
A=A_{a, b, f}(\alpha, \lambda ; h)=\left|f^{\prime}(b)\right|^{q} \int_{0}^{1-\alpha}|t-\alpha \lambda| h(t) d t+\left|f^{\prime}(a)\right|^{q} \int_{0}^{1-\alpha}|t-\alpha \lambda| h(1-t) d t, \\
B=B_{a, b, f}(\alpha, \lambda ; h)=\left|f^{\prime}(b)\right|^{q} \int_{1-\alpha}^{1}|t-1+\lambda(1-\alpha)| h(t) d t+\left|f^{\prime}(a)\right|^{q} \int_{1-\alpha}^{1}|t-1+\lambda(1-\alpha)| h(1-t) d t
\end{gathered}
$$

Proof. Suppose that $q \geq 1$. From Lemma 1 and using the well known power mean inequality, we have

$$
\begin{aligned}
\left|I_{f}(\lambda, \alpha, a, b)\right| & \leq(b-a)\left[\int_{0}^{1-\alpha}|t-\alpha \lambda|\left|f^{\prime}(t b+(1-t) a)\right| d t+\int_{1-\alpha}^{1}|t-1+\lambda(1-\alpha)|\left|f^{\prime}(t b+(1-t) a)\right| d t\right] \\
& \leq(b-a)\left\{\left(\int_{0}^{1-\alpha}|t-\alpha \lambda| d t\right)^{1-\frac{1}{q}}\left(\int_{0}^{1-\alpha}|t-\alpha \lambda|\left|f^{\prime}(t b+(1-t) a)\right|^{q} d t\right)^{\frac{1}{q}}\right. \\
& \left.+\left(\int_{1-\alpha}^{1}|t-1+\lambda(1-\alpha)| d t\right)^{1-\frac{1}{q}}\left(\int_{1-\alpha}^{1}|t-1+\lambda(1-\alpha)|\left|f^{\prime}(t b+(1-t) a)\right|^{q} d t\right)^{\frac{1}{q}}\right\}
\end{aligned}
$$

Consider

$$
I_{1}=\int_{0}^{1-\alpha}|t-\alpha \lambda|\left|f^{\prime}(t b+(1-t) a)\right|^{q} d t, \quad I_{2}=\int_{1-\alpha}^{1}|t-1+\lambda(1-\alpha)|\left|f^{\prime}(t b+(1-t) a)\right|^{q} d t
$$

Since $\left|f^{\prime}\right|^{q}$ is $h$-convex on $[a, b]$,

$$
I_{1} \leq\left|f^{\prime}(b)\right|^{q} \int_{0}^{1-\alpha}|t-\alpha \lambda| h(t) d t+\left|f^{\prime}(a)\right|^{q} \int_{0}^{1-\alpha}|t-\alpha \lambda| h(1-t) d t .
$$

Similarly

$$
I_{2} \leq\left|f^{\prime}(b)\right|^{q} \int_{1-\alpha}^{1}|t-1+\lambda(1-\alpha)| h(t) d t+\left|f^{\prime}(a)\right|^{q} \int_{1-\alpha}^{1}|t-1+\lambda(1-\alpha)| h(1-t) d t .
$$


Additionally, by simple computation

$$
\begin{gathered}
\int_{0}^{1-\alpha}|t-\alpha \lambda| d t=\left\{\begin{array}{l}
\gamma_{2}, \alpha \lambda \leq 1-\alpha \\
\gamma_{1}, \alpha \lambda \geq 1-\alpha
\end{array}\right. \\
\gamma_{1}=(1-\alpha)\left[\alpha \lambda-\frac{(1-\alpha)}{2}\right], \gamma_{2}=(\alpha \lambda)^{2}-\gamma_{1}, \\
\int_{1-\alpha}^{1}|t-1+\lambda(1-\alpha)| d t=\left\{\begin{array}{l}
v_{1}, 1-\lambda(1-\alpha) \leq 1-\alpha \\
v_{2}, 1-\lambda(1-\alpha) \geq 1-\alpha
\end{array},\right. \\
v_{1}=\frac{1-(1-\alpha)^{2}}{2}-\alpha[1-\lambda(1-\alpha)], \\
v_{2}=\frac{1+(1-\alpha)^{2}}{2}-(\lambda+1)(1-\alpha)[1-\lambda(1-\alpha)] .
\end{gathered}
$$

Thus, using (10) (13) in (9), we obtain the inequality (6). This completes the proof.

Corollary 1. Under the assumptions of Theorem 4 with $q=1$, we have

$$
\begin{aligned}
\left|I_{f}(\lambda, \alpha, a, b)\right| \leq(b-a) & \left|f^{\prime}(b)\right|\left[\int_{0}^{1-\alpha}|t-\alpha \lambda| h(t) d t+\int_{1-\alpha}^{1}|t-1+\lambda(1-\alpha)| h(t) d t\right] \\
& \left.\left|f^{\prime}(a)\right|\left[\int_{0}^{1-\alpha}|t-\alpha \lambda| h(1-t) d t+\int_{1-\alpha}^{1}|t-1+\lambda(1-\alpha)| h(1-t) d t\right]\right\}
\end{aligned}
$$

Corollary 2. Under the assumptions of Theorem 4 with $\alpha=1 / 2$, we obtain the following generalized inequality

$$
\begin{aligned}
& \left|\lambda\left(\frac{f(a)+f(b)}{2}\right)+(1-\lambda) f\left(\frac{a+b}{2}\right)-\frac{1}{b-a} \int_{a}^{b} f(x) d x\right| \\
& \leq(b-a)\left(\frac{2 \lambda^{2}-2 \lambda+1}{8}\right)^{1-1 / q}\left[A_{a, b, f}^{1 / q}(1 / 2, \lambda ; h)+B_{a, b, f}^{1 / q}(1 / 2, \lambda ; h)\right],
\end{aligned}
$$

where

$$
\begin{aligned}
& A_{a, b, f}(1 / 2, \lambda ; h)=\left|f^{\prime}(b)\right|^{q} \int_{0}^{1 / 2}\left|t-\frac{\lambda}{2}\right| h(t) d t+\left|f^{\prime}(a)\right|^{q} \int_{0}^{1 / 2}\left|t-\frac{\lambda}{2}\right| h(1-t) d t, \\
& B_{a, b, f}(1 / 2, \lambda ; h)=\left|f^{\prime}(b)\right|^{q} \int_{0}^{1 / 2}\left|t-\frac{\lambda}{2}\right| h(1-t) d t+\left|f^{\prime}(a)\right|^{q} \int_{0}^{1 / 2}\left|t-\frac{\lambda}{2}\right| h(t) d t
\end{aligned}
$$

Especially, in the inequality (14), 
(i) if we choose $\lambda=1 / 3$, we have the following Simpson type inequality

$$
\begin{aligned}
& \left|\frac{1}{3}\left[\frac{f(a)+f(b)}{2}+2 f\left(\frac{a+b}{2}\right)\right]-\frac{1}{b-a} \int_{a}^{b} f(x) d x\right| \\
& \leq(b-a)\left(\frac{5}{72}\right)^{1-1 / q}\left[A_{a, b, f}^{1 / q}(1 / 2,1 / 3 ; h)+B_{a, b, f}^{1 / q}(1 / 2,1 / 3 ; h)\right] .
\end{aligned}
$$

(ii) if we choose $\lambda=0$, we have the following midpoint inequality

$$
\left|f\left(\frac{a+b}{2}\right)-\frac{1}{b-a} \int_{a}^{b} f(x) d x\right| \leq(b-a)\left(\frac{1}{8}\right)^{1-1 / q}\left[A_{a, b, f}^{1 / q}(1 / 2,0 ; h)+B_{a, b, f}^{1 / q}(1 / 2,0 ; h)\right] .
$$

(iii) if we choose $\lambda=1$, we have the following trapezoid inequality

$$
\left|\frac{f(a)+f(b)}{2}-\frac{1}{b-a} \int_{a}^{b} f(x) d x\right| \leq(b-a)\left(\frac{1}{8}\right)^{1-1 / q}\left[A_{a, b, f}^{1 / q}(1 / 2,1 ; h)+B_{a, b, f}^{1 / q}(1 / 2,1 ; h)\right] .
$$

(iv) if we choose $\lambda=1 / 2$, we have the following inequality

$$
\begin{aligned}
& \left|\frac{1}{2}\left[\frac{f(a)+f(b)}{2}+f\left(\frac{a+b}{2}\right)\right]-\frac{1}{b-a} \int_{a}^{b} f(x) d x\right| \\
& \leq(b-a)\left(\frac{1}{16}\right)^{1-1 / q}\left[A_{a, b, f}^{1 / q}(1 / 2,1 / 2 ; h)+B_{a, b, f}^{1 / q}(1 / 2,1 / 2 ; h)\right] .
\end{aligned}
$$

Remark. In Theorem 4 if we take $h(t)=1$, we obtain the same of the inequalities in [9, Theorem 1.2].

Remark. In Theorem 4 if we take $h(t)=t$, we obtain the same of the inequalities in [7, Theorem 2.2].

Remark. In Theorem 4 if we take $I \subseteq[0, \infty)$ and $h(t)=t^{s}, s \in(0,1]$, we obtain the same of the inequalities in [8, Theorem 7].

Using Lemma 1 we shall give another result for convex functions as follows.

Theorem 5. Let $f: I \subseteq \mathbb{R} \rightarrow \mathbb{R}$ be a differentiable mapping on $I^{\circ}$ such that $f^{\prime} \in L[a, b]$, where $a, b \in I^{\circ}$ with $a<b$ and $\alpha, \lambda \in[0,1]$. If $\left|f^{\prime}\right|^{q}$ is h-convex on $[a, b], q>1$, then the following inequality holds:

$$
\begin{gathered}
\left|I_{f}(\lambda, \alpha, a, b)\right| \leq(b-a)\left(\frac{1}{p+1}\right)^{\frac{1}{p}}\left(\int_{0}^{1} h(t) d t\right)^{\frac{1}{q}} \\
\times\left\{\begin{array}{l}
{\left[\begin{array}{c}
\frac{1}{p} \\
\varepsilon_{1}^{\frac{1}{p}}(\alpha, \lambda) C_{a, b, f}^{\frac{1}{q}}(\alpha)+\varepsilon_{3}^{\frac{1}{p}}(\alpha, \lambda) D_{a, b, f}^{\frac{1}{q}}(\alpha) \\
\varepsilon_{1}^{\frac{1}{p}}(\alpha, \lambda) C_{a, b, f}^{\frac{1}{q}}(\alpha)+\varepsilon_{4}^{\frac{1}{p}}(\alpha, \lambda) D_{a, b, f}^{\frac{1}{q}}(\alpha) \\
{\left[\varepsilon_{2}^{\frac{1}{p}}(\alpha, \lambda) C_{a, b, f}^{\frac{1}{q}}(\alpha)+\varepsilon_{3}^{\frac{1}{p}}(\alpha, \lambda) D_{a, b, f}^{\frac{1}{q}}(\alpha)\right.}
\end{array}\right], \alpha \lambda \leq 1-\lambda(1-\alpha) \leq 1-\alpha \leq \alpha \lambda \leq 1-\lambda(1-\alpha)}
\end{array}\right.
\end{gathered}
$$


where

$$
\begin{gathered}
C_{a, b, f}(\alpha)=(1-\alpha)\left[\left|f^{\prime}((1-\alpha) b+\alpha a)\right|^{q}+\left|f^{\prime}(a)\right|^{q}\right], \\
D_{a, b, f}(\alpha)=\alpha\left[\left|f^{\prime}((1-\alpha) b+\alpha a)\right|^{q}+\left|f^{\prime}(b)\right|^{q}\right], \\
\varepsilon_{1}(\alpha, \lambda)=(\alpha \lambda)^{p+1}+(1-\alpha-\alpha \lambda)^{p+1}, \varepsilon_{2}(\alpha, \lambda)=(\alpha \lambda)^{p+1}-(\alpha \lambda-1+\alpha)^{p+1}, \\
\varepsilon_{3}(\alpha, \lambda)=[\lambda(1-\alpha)]^{p+1}+[\alpha-\lambda(1-\alpha)]^{p+1}, \varepsilon_{4}(\alpha, \lambda)=[\lambda(1-\alpha)]^{p+1}-[\lambda(1-\alpha)-\alpha]^{p+1},
\end{gathered}
$$

and $\frac{1}{p}+\frac{1}{q}=1$.

Proof. From Lemma 1 and by Hölder's integral inequality, we have

$$
\begin{aligned}
\left|I_{f}(\lambda, \alpha, a, b)\right| & \leq(b-a)\left[\int_{0}^{1-\alpha}|t-\alpha \lambda|\left|f^{\prime}(t b+(1-t) a)\right| d t+\int_{1-\alpha}^{1}|t-1+\lambda(1-\alpha)|\left|f^{\prime}(t b+(1-t) a)\right| d t\right] \\
& \leq(b-a)\left\{\left(\int_{0}^{1-\alpha}|t-\alpha \lambda|^{p} d t\right)^{\frac{1}{p}}\left(\int_{0}^{1-\alpha}\left|f^{\prime}(t b+(1-t) a)\right|^{q} d t\right)^{\frac{1}{q}}\right. \\
& \left.+\left(\int_{1-\alpha}^{1}|t-1+\lambda(1-\alpha)|^{p} d t\right)^{\frac{1}{p}}\left(\int_{1-\alpha}^{1}\left|f^{\prime}(t b+(1-t) a)\right|^{q} d t\right)^{\frac{1}{q}}\right\} .
\end{aligned}
$$

Since $\left|f^{\prime}\right|^{q}$ is $h$-convex on $[a, b]$, for $\alpha \in[0,1)$ by the inequality (3), we get

$$
\begin{aligned}
\int_{0}^{1-\alpha}\left|f^{\prime}(t b+(1-t) a)\right|^{q} d t & =(1-\alpha)\left[\frac{1}{(1-\alpha)(b-a)} \int_{a}^{(1-\alpha) b+\alpha a}\left|f^{\prime}(x)\right|^{q} d x\right] \\
& \leq(1-\alpha)\left[\left|f^{\prime}((1-\alpha) b+\alpha a)\right|^{q}+\left|f^{\prime}(a)\right|^{q}\right] \int_{0}^{1} h(t) d t .
\end{aligned}
$$

The inequality (18) also holds for $\alpha=1$. Similarly, for $\alpha \in(0,1]$ by the inequality (3), we have

$$
\begin{aligned}
\int_{1-\alpha}^{1}\left|f^{\prime}(t b+(1-t) a)\right|^{q} d t & =\alpha\left[\frac{1}{\alpha(b-a)} \int_{(1-\alpha) b+\alpha a}^{b}\left|f^{\prime}(x)\right|^{q} d x\right] \\
& \leq \alpha\left[\left|f^{\prime}((1-\alpha) b+\alpha a)\right|^{q}+\left|f^{\prime}(b)\right|^{q}\right] \int_{0}^{1} h(t) d t .
\end{aligned}
$$

The inequality (19) also holds for $\alpha=0$. By simple computation

$$
\int_{0}^{1-\alpha}|t-\alpha \lambda|^{p} d t=\left\{\begin{array}{l}
\frac{(\alpha \lambda)^{p+1}+(1-\alpha-\alpha \lambda)^{p+1}}{p+1}, \alpha \lambda \leq 1-\alpha \\
\frac{(\alpha \lambda)^{p+1}-(\alpha \lambda-1+\alpha)^{p+1}}{p+1}, \alpha \lambda \geq 1-\alpha
\end{array}\right.
$$


and

$$
\int_{1-\alpha}^{1}|t-1+\lambda(1-\alpha)|^{p} d t=\left\{\begin{array}{l}
\frac{[\lambda(1-\alpha)]^{p+1}+[\alpha-\lambda(1-\alpha)]^{p+1}}{p+1}, 1-\alpha \leq 1-\lambda(1-\alpha) \\
\frac{[\lambda(1-\alpha)]^{p+1}-[\lambda(1-\alpha)-\alpha]^{p+1}}{p+1}, 1-\alpha \geq 1-\lambda(1-\alpha)
\end{array},\right.
$$

thus, using (18)-(21) in (17), we obtain the inequality (15). This completes the proof.

Corollary 3. Under the assumptions of Theorem 5 with $\alpha=1 / 2$, we obtain the following generalized inequality

$$
\begin{aligned}
& \left|\lambda\left(\frac{f(a)+f(b)}{2}\right)+(1-\lambda) f\left(\frac{a+b}{2}\right)-\frac{1}{b-a} \int_{a}^{b} f(x) d x\right| \\
& \leq \frac{(b-a)}{4}\left(\int_{0}^{1} h(t) d t\right)^{\frac{1}{q}}\left(\frac{\lambda^{p+1}+(1-\lambda)^{p+1}}{p+1}\right)^{\frac{1}{p}} \times\left(\left[\left|f^{\prime}\left(\frac{a+b}{2}\right)\right|^{q}+\left|f^{\prime}(a)\right|^{q}\right]^{1 / q}+\left[\left|f^{\prime}\left(\frac{a+b}{2}\right)\right|^{q}+\left|f^{\prime}(b)\right|^{q}\right]^{1 / q}\right)
\end{aligned}
$$

which is identical to inequality (4).

Especially, in the inequality (22),

(i) if we choose $\lambda=1 / 3$, we have the following Simpson type inequality

$$
\begin{aligned}
& \left|\frac{1}{3}\left[\frac{f(a)+f(b)}{2}+2 f\left(\frac{a+b}{2}\right)\right]-\frac{1}{b-a} \int_{a}^{b} f(x) d x\right| \\
& \leq \frac{(b-a)}{12}\left(\int_{0}^{1} h(t) d t\right)^{\frac{1}{q}}\left(\frac{1+2^{p+1}}{3(p+1)}\right)^{\frac{1}{p}} \\
& \times\left(\left[\left|f^{\prime}\left(\frac{a+b}{2}\right)\right|^{q}+\left|f^{\prime}(a)\right|^{q}\right]^{1 / q}+\left[\left|f^{\prime}\left(\frac{a+b}{2}\right)\right|^{q}+\left|f^{\prime}(b)\right|^{q}\right]^{1 / q}\right) .
\end{aligned}
$$

(ii) if we choose $\lambda=0$, we have the following midpoint inequality

$$
\begin{aligned}
& \left|f\left(\frac{a+b}{2}\right)-\frac{1}{b-a} \int_{a}^{b} f(x) d x\right| \leq \frac{(b-a)}{4}\left(\int_{0}^{1} h(t) d t\right)^{1 / q}\left(\frac{1}{p+1}\right)^{1 / p} \\
& \left.\times\left(\left.|| f^{\prime}\left(\frac{a+b}{2}\right)\right|^{q}+\left|f^{\prime}(a)\right|^{q}\right]^{1 / q}+\left[\left|f^{\prime}\left(\frac{a+b}{2}\right)\right|^{q}+\left|f^{\prime}(b)\right|^{q}\right]^{1 / q}\right) .
\end{aligned}
$$

(iii) if we choose $\lambda=1$, we have the following trapezoid inequality

$$
\begin{aligned}
& \left|\frac{f(a)+f(b)}{2}-\frac{1}{b-a} \int_{a}^{b} f(x) d x\right| \leq \frac{(b-a)}{4}\left(\int_{0}^{1} h(t) d t\right)^{1 / q}\left(\frac{1}{p+1}\right)^{1 / p} \\
& \times\left(\left[\left|f^{\prime}\left(\frac{a+b}{2}\right)\right|^{q}+\left|f^{\prime}(a)\right|^{q}\right]^{1 / q}+\left[\left|f^{\prime}\left(\frac{a+b}{2}\right)\right|^{q}+\left|f^{\prime}(b)\right|^{q}\right]^{1 / q}\right) .
\end{aligned}
$$


(iv) if we choose $\lambda=1 / 2$, we have the following inequality

$$
\begin{aligned}
& \left|\frac{1}{2}\left[\frac{f(a)+f(b)}{2}+f\left(\frac{a+b}{2}\right)\right]-\frac{1}{b-a} \int_{a}^{b} f(x) d x\right| \\
& \leq \frac{(b-a)}{8}\left(\int_{0}^{1} h(t) d t\right)^{1 / q}\left(\frac{1}{p+1}\right)^{1 / p} \times\left(\left[\left|f^{\prime}\left(\frac{a+b}{2}\right)\right|^{q}+\left|f^{\prime}(a)\right|^{q}\right]^{1 / q}+\left[\left|f^{\prime}\left(\frac{a+b}{2}\right)\right|^{q}+\left|f^{\prime}(b)\right|^{q}\right]^{1 / q}\right) .
\end{aligned}
$$

Remark. In Theorem 5 if we take $h(t)=1$, we obtain the same of the inequalities in [9, Theorem 1.3].

Remark. In Theorem 5 if we take $h(t)=t$, we obtain the same of the inequalities in [7, Theorem 2.8].

Remark. In Theorem 5 if we take $I \subseteq[0, \infty)$ and $h(t)=t^{s}, s \in(0,1]$, we obtain the same of the inequalities in [8, Theorem 7].

Theorem 6. Let $f: I \subseteq \mathbb{R} \rightarrow \mathbb{R}$ be a differentiable mapping on $I^{\circ}$ such that $f^{\prime} \in L[a, b]$, where $a, b \in I^{\circ}$ with $a<b$ and $\alpha, \lambda \in[0,1]$. If $\left|f^{\prime}\right|^{q}$ is $h$-concave on $[a, b], q>1$, then the following inequality holds:

$$
\begin{gathered}
\left|I_{f}(\lambda, \alpha, a, b)\right| \leq(b-a)\left(\frac{1}{2 h\left(\frac{1}{2}\right)}\right)^{\frac{1}{q}}\left(\frac{1}{p+1}\right)^{\frac{1}{p}} \\
\times\left\{\begin{array}{c}
{\left[\varepsilon_{1}^{\frac{1}{p}}(\alpha, \lambda) E_{a, b, f}^{\frac{1}{q}}(\alpha)+\varepsilon_{3}^{\frac{1}{p}}(\alpha, \lambda) F_{a, b, f}^{\frac{1}{q}}(\alpha)\right], \alpha \lambda \leq 1-\alpha \leq 1-\lambda(1-\alpha)} \\
{\left[\varepsilon_{1}^{\frac{1}{p}}(\alpha, \lambda) E_{a, b, f}^{\frac{1}{q}}(\alpha)+\varepsilon_{4}^{\frac{1}{p}}(\alpha, \lambda) F_{a, b, f}^{\frac{1}{q}}(\alpha)\right], \alpha \lambda \leq 1-\lambda(1-\alpha) \leq 1-\alpha,} \\
\left.-\varepsilon_{2}^{\frac{1}{p}}(\alpha, \lambda) E_{a, b, f}^{\frac{1}{q}}(\alpha)+\varepsilon_{3}^{\frac{1}{p}}(\alpha, \lambda) F_{a, b, f}^{\frac{1}{q}}(\alpha)\right], 1-\alpha \leq \alpha \lambda \leq 1-\lambda(1-\alpha)
\end{array}\right.
\end{gathered}
$$

where $1 / p+1 / q=1$,

$$
E_{a, b, f}(\alpha)=(1-\alpha)\left|f^{\prime}\left(\frac{(1-\alpha) b+(1+\alpha) a}{2}\right)\right|^{q}, F_{a, b, f}(\alpha)=\alpha\left|f^{\prime}\left(\frac{(2-\alpha) b+\alpha a}{2}\right)\right|^{q}
$$

and $\varepsilon_{1}, \varepsilon_{2}, \varepsilon_{3}, \varepsilon_{4}$ are defined as in (16).

Proof. We proceed similarly as in the proof Theorem 5. Since $\left|f^{\prime}\right|^{q}$ is $h$-concave on $[a, b]$, for $\alpha \in[0,1)$ by the inequality (3), we get

$$
\begin{aligned}
\int_{0}^{1-\alpha}\left|f^{\prime}(t b+(1-t) a)\right|^{q} d t & =(1-\alpha)\left[\frac{1}{(1-\alpha)(b-a)} \int_{a}^{(1-\alpha) b+\alpha a}\left|f^{\prime}(x)\right|^{q} d x\right] \\
& \leq \frac{(1-\alpha)}{2 h\left(\frac{1}{2}\right)}\left|f^{\prime}\left(\frac{(1-\alpha) b+(1+\alpha) a}{2}\right)\right|^{q}
\end{aligned}
$$

The inequality (24) also holds for $\alpha=1$. Similarly, for $\alpha \in(0,1]$ by the inequality (3), we have

$$
\int_{1-\alpha}^{1}\left|f^{\prime}(t b+(1-t) a)\right|^{q} d t=\alpha\left[\frac{1}{\alpha(b-a)} \int_{(1-\alpha) b+\alpha a}^{b}\left|f^{\prime}(x)\right|^{q} d x\right] \leq \frac{\alpha}{2 h\left(\frac{1}{2}\right)}\left|f^{\prime}\left(\frac{(2-\alpha) b+\alpha a}{2}\right)\right|^{q}
$$


The inequality (25) also holds for $\alpha=0$. Thus, using (20),(21),(24)and (25) in (17), we obtain the inequality (23). This completes the proof.

Corollary 4. Under the assumptions of Theorem 6 with $\alpha=1 / 2$, we obtain the following generalized inequality

$$
\begin{aligned}
& \left|\lambda\left(\frac{f(a)+f(b)}{2}\right)+(1-\lambda) f\left(\frac{a+b}{2}\right)-\frac{1}{b-a} \int_{a}^{b} f(x) d x\right| \\
& \leq \frac{(b-a)}{4}\left(\frac{1}{2 h\left(\frac{1}{2}\right)}\right)^{\frac{1}{q}}\left(\frac{\lambda^{p+1}+(1-\lambda)^{p+1}}{p+1}\right)^{\frac{1}{p}}\left[\left|f^{\prime}\left(\frac{3 a+b}{4}\right)\right|+\left|f^{\prime}\left(\frac{3 b+a}{4}\right)\right|\right]
\end{aligned}
$$

which is identical to inequality (5).

Especially, in the inequality (26),

(i) if we choose $\lambda=1 / 3$, we have the following Simpson type inequality

$$
\begin{aligned}
& \left|\frac{1}{3}\left[\frac{f(a)+f(b)}{2}+2 f\left(\frac{a+b}{2}\right)\right]-\frac{1}{b-a} \int_{a}^{b} f(x) d x\right| \\
& \leq \frac{(b-a)}{12}\left(\frac{1}{2 h\left(\frac{1}{2}\right)}\right)^{\frac{1}{q}}\left(\frac{1+2^{p+1}}{3(p+1)}\right)^{\frac{1}{p}}\left[\left|f^{\prime}\left(\frac{3 a+b}{4}\right)\right|+\left|f^{\prime}\left(\frac{3 b+a}{4}\right)\right|\right] .
\end{aligned}
$$

(ii) if we choose $\lambda=0$, we have the following midpoint inequality which is the same the inequality (2.9) in [14, Corollary 3].

$$
\left|f\left(\frac{a+b}{2}\right)-\frac{1}{b-a} \int_{a}^{b} f(x) d x\right| \leq \frac{(b-a)}{4}\left(\frac{1}{2 h\left(\frac{1}{2}\right)}\right)^{\frac{1}{q}}\left(\frac{1}{p+1}\right)^{\frac{1}{p}}\left[\left|f^{\prime}\left(\frac{3 a+b}{4}\right)\right|+\left|f^{\prime}\left(\frac{3 b+a}{4}\right)\right|\right] .
$$

(iii) if we choose $\lambda=1$, we have the following trapezoid inequality

$$
\left|\frac{f(a)+f(b)}{2}-\frac{1}{b-a} \int_{a}^{b} f(x) d x\right| \leq \frac{(b-a)}{4}\left(\frac{1}{2 h\left(\frac{1}{2}\right)}\right)^{\frac{1}{q}}\left(\frac{1}{p+1}\right)^{\frac{1}{p}}\left[\left|f^{\prime}\left(\frac{3 a+b}{4}\right)\right|+\left|f^{\prime}\left(\frac{3 b+a}{4}\right)\right|\right] .
$$

(iv) if we choose $\lambda=1 / 2$, we have the following inequality

$$
\begin{aligned}
& \left|\frac{1}{2}\left[\frac{f(a)+f(b)}{2}+f\left(\frac{a+b}{2}\right)\right]-\frac{1}{b-a} \int_{a}^{b} f(x) d x\right| \\
& \leq \frac{(b-a)}{8}\left(\frac{1}{2 h\left(\frac{1}{2}\right)}\right)^{\frac{1}{q}}\left(\frac{1}{p+1}\right)^{\frac{1}{p}}\left[\left|f^{\prime}\left(\frac{3 a+b}{4}\right)\right|+\left|f^{\prime}\left(\frac{3 b+a}{4}\right)\right|\right] .
\end{aligned}
$$

Corollary 5. Under the assumptions of Theorem 6 with $I \subseteq[0, \infty), h(t)=t^{s}, s \in(0,1]$, we have

$$
\left|I_{f}(\lambda, \alpha, a, b)\right| \leq(b-a) 2^{\frac{s-1}{q}}\left(\frac{1}{p+1}\right)^{\frac{1}{p}}
$$




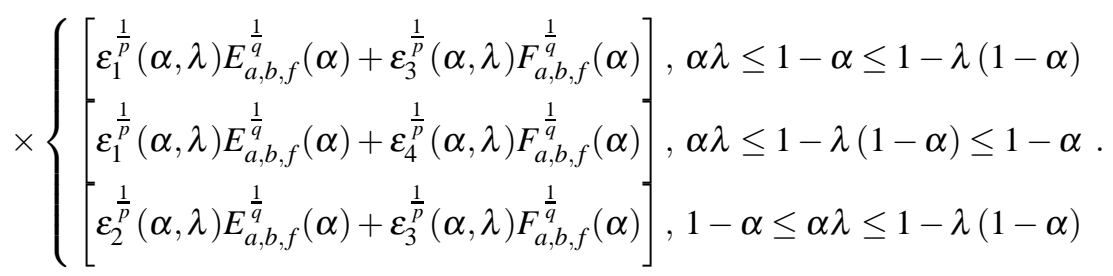

Corollary 6. Under the assumptions of Theorem 6 with $h(t)=t$, we have

$$
\begin{gathered}
\left|I_{f}(\lambda, \alpha, a, b)\right| \leq(b-a)\left(\frac{1}{p+1}\right)^{\frac{1}{p}} \\
\times\left\{\begin{array}{c}
{\left[\varepsilon_{1}^{\frac{1}{p}}(\alpha, \lambda) E_{a, b, f}^{\frac{1}{q}}(\alpha)+\varepsilon_{3}^{\frac{1}{p}}(\alpha, \lambda) F_{a, b, f}^{\frac{1}{q}}(\alpha)\right.} \\
\varepsilon_{1}^{\frac{1}{p}}(\alpha, \lambda) E_{a, b, f}^{\frac{1}{q}}(\alpha)+\varepsilon_{4}^{\frac{1}{p}}(\alpha, \lambda) F_{a, b, f}^{\frac{1}{q}}(\alpha) \\
{\left[\begin{array}{l}
\frac{1}{p} \\
\varepsilon_{2}^{\frac{1}{q}}(\alpha, \lambda) E_{a, b, f}^{\frac{1}{q}}(\alpha)+\varepsilon_{3}^{\frac{1}{p}}(\alpha, \lambda) F_{a, b, f}^{\frac{1}{q}}(\alpha)
\end{array}\right], \alpha \lambda \leq 1-\lambda(1-\alpha) \leq 1-\alpha . \alpha \leq \alpha \leq 1-\lambda(1-\alpha)}
\end{array}\right.
\end{gathered}
$$

Corollary 7. Under the assumptions of Theorem 6 with $h(t)=1$, we have

$$
\begin{gathered}
\left|I_{f}(\lambda, \alpha, a, b)\right| \leq(b-a) 2^{-\frac{1}{q}}\left(\frac{1}{p+1}\right)^{\frac{1}{p}} \\
\times\left\{\begin{array}{c}
{\left[\varepsilon_{1}^{\frac{1}{p}}(\alpha, \lambda) E_{a, b, f}^{\frac{1}{q}}(\alpha)+\varepsilon_{3}^{\frac{1}{p}}(\alpha, \lambda) F_{a, b, f}^{\frac{1}{q}}(\alpha)\right.} \\
\varepsilon_{1}^{\frac{1}{p}}(\alpha, \lambda) E_{a, b, f}^{\frac{1}{q}}(\alpha)+\varepsilon_{4}^{\frac{1}{p}}(\alpha, \lambda) F_{a, b, f}^{\frac{1}{q}}(\alpha) \\
{\left[\begin{array}{l}
\frac{1}{p} \\
\varepsilon_{2}^{\frac{1}{q}}(\alpha, \lambda) E_{a, b, f}^{\frac{1}{q}}(\alpha)+\varepsilon_{3}^{\frac{1}{p}}(\alpha, \lambda) F_{a, b, f}^{\frac{1}{q}}(\alpha)
\end{array}\right], \alpha \lambda \leq 1-\lambda(1-\alpha) \leq 1-\alpha . \alpha \leq \alpha \leq 1-\lambda(1-\alpha)}
\end{array}\right.
\end{gathered}
$$

Corollary 8. Under the assumptions of Theorem 6 with $h(t)=\frac{1}{t}, t \in(0,1)$, we have

$$
\begin{aligned}
& \left|\lambda(\alpha f(a)+(1-\alpha) f(b))+(1-\lambda) f(\alpha a+(1-\alpha) b)-\frac{1}{b-a} \int_{a}^{b} f(x) d x\right| \leq \frac{b-a}{4^{\frac{1}{q}}(p+1)^{1 / p}} \\
& \times\left\{\begin{array}{l}
{\left[\varepsilon_{1}^{\frac{1}{p}}(\alpha, \lambda) E_{a, b, f}^{\frac{1}{q}}(\alpha)+\varepsilon_{3}^{\frac{1}{p}}(\alpha, \lambda) F_{a, b, f}^{\frac{1}{q}}(\alpha)\right], \alpha \lambda \leq 1-\alpha \leq 1-\lambda(1-\alpha)} \\
{\left[\varepsilon_{1}^{\frac{1}{p}}(\alpha, \lambda) E_{a, b, f}^{\frac{1}{q}}(\alpha)+\varepsilon_{4}^{\frac{1}{p}}(\alpha, \lambda) F_{a, b, f}^{\frac{1}{q}}(\alpha)\right], \alpha \lambda \leq 1-\lambda(1-\alpha) \leq 1-\alpha} \\
{\left[\varepsilon_{2}^{\frac{1}{p}}(\alpha, \lambda) E_{a, b, f}^{\frac{1}{q}}(\alpha)+\varepsilon_{3}^{\frac{1}{p}}(\alpha, \lambda) F_{a, b, f}^{\frac{1}{q}}(\alpha)\right], 1-\alpha \leq \alpha \lambda \leq 1-\lambda(1-\alpha)}
\end{array}\right.
\end{aligned}
$$

Remark. In Corollary 5, if we take $\alpha=\frac{1}{2}$ and $\lambda=1$, then we have the following trapezoid inequality

$$
\left|\frac{f(a)+f(b)}{2}-\frac{1}{b-a} \int_{a}^{b} f(x) d x\right| \leq \frac{b-a}{4}\left(\frac{1}{p+1}\right)^{\frac{1}{p}}\left(\frac{1}{2}\right)^{\frac{1-s}{q}}\left[\left|f^{\prime}\left(\frac{3 b+a}{4}\right)\right|+\left|f^{\prime}\left(\frac{3 a+b}{4}\right)\right|\right]
$$

which is the same of the inequality in [12, Theorem 8 (i)]. 
Remark. In Corollary 5, if we take $\alpha=\frac{1}{2}$ and $\lambda=0$, then we have the following midpoint inequality

$$
\left|f\left(\frac{a+b}{2}\right)-\frac{1}{b-a} \int_{a}^{b} f(x) d x\right| \leq \frac{b-a}{4}\left(\frac{1}{p+1}\right)^{\frac{1}{p}}\left(\frac{1}{2}\right)^{\frac{1-s}{q}}\left[\left|f^{\prime}\left(\frac{3 b+a}{4}\right)\right|+\left|f^{\prime}\left(\frac{3 a+b}{4}\right)\right|\right]
$$

which is the same of the inequality in [12, Theorem 8 (ii)].

Remark. In Corollary 6 , if we take $\alpha=\frac{1}{2}$ and $\lambda=1$, then we have the following trapezoid inequality

$$
\left|\frac{f(a)+f(b)}{2}-\frac{1}{b-a} \int_{a}^{b} f(x) d x\right| \leq \frac{b-a}{4}\left(\frac{1}{p+1}\right)^{\frac{1}{p}}\left[\left|f^{\prime}\left(\frac{3 b+a}{4}\right)\right|+\left|f^{\prime}\left(\frac{3 a+b}{4}\right)\right|\right]
$$

which is the same of the inequality in [11, Theorem 2].

Remark. In Corollary 6, if we take $\alpha=\frac{1}{2}$ and $\lambda=0$, then we have the following trapezoid inequality

$$
\left|f\left(\frac{a+b}{2}\right)-\frac{1}{b-a} \int_{a}^{b} f(x) d x\right| \leq \frac{b-a}{4}\left(\frac{1}{p+1}\right)^{\frac{1}{p}}\left[\left|f^{\prime}\left(\frac{3 b+a}{4}\right)\right|+\left|f^{\prime}\left(\frac{3 a+b}{4}\right)\right|\right]
$$

which is the same of the inequality in [1, Theorem 2.5].

\section{Some applications for special means}

Let us recall the following special means of arbitrary real numbers $a, b$ with $a \neq b$ and $\alpha \in[0,1]$ :

1.The unweighted arithmetic mean

$$
A=A(a, b):=\frac{a+b}{2}, a, b \geq 0
$$

2.The weighted geometric mean

$$
G_{\alpha}(a, b)=a^{\alpha} b^{1-\alpha}, a, b>0 .
$$

3.The unweighted geometric mean

$$
G=G(a, b)=\sqrt{a b}, a, b>0 .
$$

4.The unweighted harmonic mean

$$
H(a, b):=\frac{2 a b}{a+b}, a, b>0 .
$$

5.Then n-Logarithmic mean

$$
L_{n}(a, b):=\left(\frac{b^{n+1}-a^{n+1}}{(n+1)(b-a)}\right)^{\frac{1}{n}}, n \in \mathbb{R} \backslash\{-1,0\}, a, b>0, a \neq b .
$$

6.The identric mean

$$
I=I(a, b)=\frac{1}{e}\left(\frac{b^{b}}{a^{a}}\right)^{\frac{1}{b-a}}, a, b>0, a \neq b
$$

Let $h$ be a nonnegative function such that $h(\alpha) \geq \alpha$ for all $\alpha \in(0,1)$ and $f(t)=t^{\frac{n}{q}+1}, t \in[a, b], a, b \in \mathbb{R}$ with $0<a<b$, $q \geq 1, n \in(-\infty, 0] \cup[1, \infty)$, then the function $\left|f^{\prime}(t)\right|^{q}=\left(\frac{n}{q}+1\right)^{q} t^{n}$ is a convex, and also is a $h$-convex according to Remark 1. Similarly, let $f(t)=\ln t, t \in[a, b], a, b \in \mathbb{R}$ with $0<a<b$, then the function $\left|f^{\prime}(t)\right|^{q}=t^{-q}, q \geq 1$, is a $h$-convex. 
Proposition 1. Let $a, b \in \mathbb{R}$ with $0<a<b, q \geq 1, n \geq 1$, and $h$ be a nonnegative function such that $h(\alpha) \geq \alpha$ for all $\alpha \in(0,1)$, then we have the following inequality:

$$
\begin{aligned}
& \left|\lambda A\left(a^{\frac{n}{q}+1}, b^{\frac{n}{q}+1}\right)+(1-\lambda) A^{\frac{n}{q}+1}(a, b)-L_{\frac{n}{q}+1}^{\frac{n}{q}+1}(a, b)\right| \\
& \leq \frac{(b-a)(n+q)}{q}\left(\frac{2 \lambda^{2}-2 \lambda+1}{8}\right)^{1-1 / q}\left[A_{a, b, *}^{1 / q}(1 / 2, \lambda ; h)+B_{a, b, *}^{1 / q}(1 / 2, \lambda ; h)\right]
\end{aligned}
$$

where

$$
\begin{aligned}
& A_{a, b, *}(1 / 2, \lambda ; h)=b^{n} \int_{0}^{1 / 2}\left|t-\frac{\lambda}{2}\right| h(t) d t+a^{n} \int_{0}^{1 / 2}\left|t-\frac{\lambda}{2}\right| h(1-t) d t, \\
& B_{a, b, *}(1 / 2, \lambda ; h)=b^{n} \int_{0}^{1 / 2}\left|t-\frac{\lambda}{2}\right| h(1-t) d t+a^{n} \int_{0}^{1 / 2}\left|t-\frac{\lambda}{2}\right| h(t) d t .
\end{aligned}
$$

Proof. The assertion follows from applied the inequality (14) to the function $f(t)=t^{\frac{n}{q}+1}, t \in[a, b]$.

Proposition 2. Let $a, b \in \mathbb{R}$ with $0<a<b, q>1, n \geq 1$, and $h$ be a nonnegative function such that $h(\alpha) \geq \alpha$ for all $\alpha \in(0,1)$, then we have the following inequality:

$$
\begin{aligned}
& \left|\lambda A\left(a^{\frac{n}{q}+1}, b^{\frac{n}{q}+1}\right)+(1-\lambda) A^{\frac{n}{q}+1}(a, b)-L_{\frac{n}{q}+1}^{\frac{n}{q}+1}(a, b)\right| \leq \frac{(b-a)(n+q)}{4 q} \\
& \times\left(\int_{0}^{1} h(t) d t\right)^{\frac{1}{q}}\left(\frac{\lambda^{p+1}+(1-\lambda)^{p+1}}{p+1}\right)^{\frac{1}{p}}\left(A^{1 / q}\left(A^{n}(a, b), a^{n}\right)+A^{1 / q}\left(A^{n}(a, b), b^{n}\right)\right) .
\end{aligned}
$$

Proof. The assertion follows from applied the inequality (22) to the function $f(t)=t^{\frac{n}{q}+1}, t \in[a, b]$.

Proposition 3. Let $a, b \in \mathbb{R}$ with $0<a<b, q \geq 1, n \geq 1$, and $h$ be a nonnegative function such that $h(\alpha) \geq \alpha$ for all $\alpha \in(0,1)$, then we have the following inequality:

$$
\left|\ln \left(\frac{G_{\lambda}(G, A)}{I}\right)\right| \leq(b-a)\left(\frac{2 \lambda^{2}-2 \lambda+1}{8}\right)^{1-1 / q}\left[A_{a, b, \bullet}^{1 / q}(1 / 2, \lambda ; h)+B_{a, b, \bullet}^{1 / q}(1 / 2, \lambda ; h)\right]
$$

where

$$
\begin{aligned}
& A_{a, b, \bullet}(1 / 2, \lambda ; h)=b^{-q} \int_{0}^{1 / 2}\left|t-\frac{\lambda}{2}\right| h(t) d t+a^{-q} \int_{0}^{1 / 2}\left|t-\frac{\lambda}{2}\right| h(1-t) d t, \\
& B_{a, b, \bullet}(1 / 2, \lambda ; h)=b^{-q} \int_{0}^{1 / 2}\left|t-\frac{\lambda}{2}\right| h(1-t) d t+a^{-q} \int_{0}^{1 / 2}\left|t-\frac{\lambda}{2}\right| h(t) d t .
\end{aligned}
$$

Proof. The assertion follows from applied the inequality (14) to the function $f(t)=\ln t, t \in[a, b]$.

Proposition 4. Let $a, b \in \mathbb{R}$ with $0<a<b, q>1, n \geq 1$, and $h$ be a nonnegative function such that $h(\alpha) \geq \alpha$ for all $\alpha \in(0,1)$, then we have the following inequality: 


$$
\left|\ln \left(\frac{G_{\lambda}(G, A)}{I}\right)\right| \leq \frac{(b-a)}{2}\left(2 \cdot \int_{0}^{1} h(t) d t\right)^{\frac{1}{q}}\left(\frac{\lambda^{p+1}+(1-\lambda)^{p+1}}{p+1}\right)^{\frac{1}{p}} \times H^{-1}\left(H^{1 / q}\left(A^{q}, a^{q}\right), H^{1 / q}\left(A^{q}, b^{q}\right)\right) .
$$

Proof. The assertion follows from applied the inequality (22) to the function $f(t)=\ln t, t \in[a, b]$.

\section{4 conclusion}

The paper deals with general integral inequalities containing all of Hermite-Hadamard and Simpson type inequalities for $h$-convex and $h$-concave functions. Firstly, some theorems on general integral inequalities are given. Later, several results of this general integral inequalities are mentioned. Some applications to special means of real numbers are also given

\section{References}

[1] M.W. Alomari, M. Darus, U.S. Kirmaci, Some inequalities of Hermite-Hadamard type for s-convex functions, Acta Math. Sci. 31B (4) (2011), 1643-1652.

[2] M. Bombardelli, S. Varošanec, Properties of $h$-convex functions related to the Hermite-Hadamard-Fejér inequalities, Comput. Math. Appl. 58 (2009), 1869-1877.

[3] W.W. Breckner, Stetigkeitsaussagen für eine Klasse verallgemeinerter konvexer funktionen in topologischen linearen Räumen, Publ. Inst. Math. 23 (1978), 13-20.

[4] S.S. Dragomir, J. Pečarić, L.E. Persson, Some inequalities of Hadamard type, Soochow J. Math. 21 (1995), 335-341.

[5] E.K. Godunova, V.I. Levin, Neravenstva dlja funkcii širokogo klassa, soderžaščego vypuklye, monotonnye i neko-torye drugie vidy funkcii, in: Vyčislitel. Mat. i. Mat. Fiz. Mežvuzov. Sb. Nauč. Trudov, pp. 138-142, MGPI, Moskva, 1985.

[6] H. Hudzik and L. Maligranda, Some remarks on s-convex functions, Aequationes Math. 48(1) (1998), 100-111.

[7] İ. İşcan, A new generalization of some integral inequalities and their applications, International Journal of Engineering and Applied sciences, 3(3) (2013), 17-27.

[8] İ. İşcan, E. Set and M. Emin Özdemir, On new general integral inequalities for s-convex functions, Appl. Math. Comput. 246 (2014) 306-315.

[9] İ. İşcan, E. Set, M. Emin Özdemir, Some new general integral inequalities for P-functions, Malaya J. Mat. 2(4)(2014) 510-516.

[10] İ. İşcan, Some new general integral inequalities for $h$-convex and $h$-concave functions, Adv. Pure Appl. Math. 5(1) (2014), $21-29$. Doi: 10.1515/apam-2013-0029.

[11] U.S. Kirmaci, M.K. Bakula, M.E. Özdemir, J. Pečarić, Hadamard-type inequalities for $s$-convex functions, Appl. Math. Comput. 193 (2007), 26-35.

[12] J. Park, Hermite-Hadamard-type inequalities for real $\alpha$-star $s$-convex mappings, J. Appl. Math. \& Informatics, 28(5-6) (2010), 1507-1518.

[13] M.Z. Sarikaya , A. Saglam and H. Yıldırım, On some Hadamard-type inequalities for $h$-convex functions, J. Math. Inequal. 2(3) (2008), 335-341.

[14] M. Tunç, Ostrowski-type inequalities via $h$-convex functions with applications to special means, J. Inequal. Appl. 2013(326) (2013), 1-10.

[15] S. Varošanec, On h-convexity, J. Math. Anal. Appl. 326 (2007), 303-311. 